\title{
Is the wind of the Oe star HD 155806 magnetically confined?
}

\author{
Alex W. Fullerton ${ }^{1}$, Véronique Petit ${ }^{2}$, Stefano Bagnulo ${ }^{3}$, \\ Gregg A. Wade ${ }^{4}$, and the MiMeS Collaboration \\ ${ }^{1}$ Space Telescope Science Institute, 3700 San Martin Drive, Baltimore, MD 21218, USA \\ email: fullerton@stsci.edu \\ ${ }^{2}$ Dept. of Geology \& Astronomy, West Chester University, West Chester, PA 19383, USA \\ email: VPetit@wcupa.edu \\ ${ }^{3}$ Armagh Observatory, College Hill, Armagh, BT61 9DG, Northern Ireland \\ email: sba@arm.ac.uk \\ ${ }^{4}$ Dept. of Physics, Royal Military College of Canada, Kingston, ON, K7K 4B4, Canada \\ email: Gregg.Wade@rmc.ca
}

\begin{abstract}
Spectropolarimetric observations of HD 155806 - the hottest Galactic Oe star - were obtained with CFHT/ESPaDOnS to test the hypothesis that disk signatures in its spectrum are due to magnetic channeling and confinement of its stellar wind. We did not detect a dipole field of sufficient strength to confine the wind, and could not confirm previous reports of a magnetic detection. It appears that stellar magnetism is not responsible for producing the disk of HD 155806.
\end{abstract}

Keywords. stars: early-type, stars: emission-line, Be, stars: winds, outflows, stars: magnetic fields, stars: individual (HD 155806)

\section{Why Are There Oe Stars?}

Oe stars are a rare subset of the O-type stars that exhibit double-peaked or central emission in their Balmer lines. This emission-line morphology is distinct from signatures of stellar winds, and is conventionally attributed to a circumstellar disk. Although Oe stars are usually considered to be a continuation of the Be phenomenon toward hotter spectral types, it is difficult to understand how stable disks can coexist with the increasingly strong stellar winds typical of O-type stars.

A plausible explanation is that the disk is maintained by a large-scale (e.g., dipolar) magnetic field that channels outflowing wind material toward the magnetic equator, in which case the disk and the wind are really a single entity. A straightforward test of this hypothesis is to search for dipolar magnetic fields of sufficient strength to confine the wind. Since HD 155806 is the hottest Galactic Oe star currently known (O7.5 V[n]e according to Walborn 1973), it should have the strongest wind; and would therefore require the largest magnetic field to confine it. Moreover, its comparatively narrow photospheric lines and brightness enhance the detectability of large-scale magnetic fields. The hypothesis of magnetic confinement was bolstered when Hubrig et al. (2007) reported the detection of a magnetic field with longitudinal strength of $\left\langle B_{z}\right\rangle=-155 \pm 37 \mathrm{G}$, though Hubrig et al. (2008) did not detect a significant field in subsequent observations. 


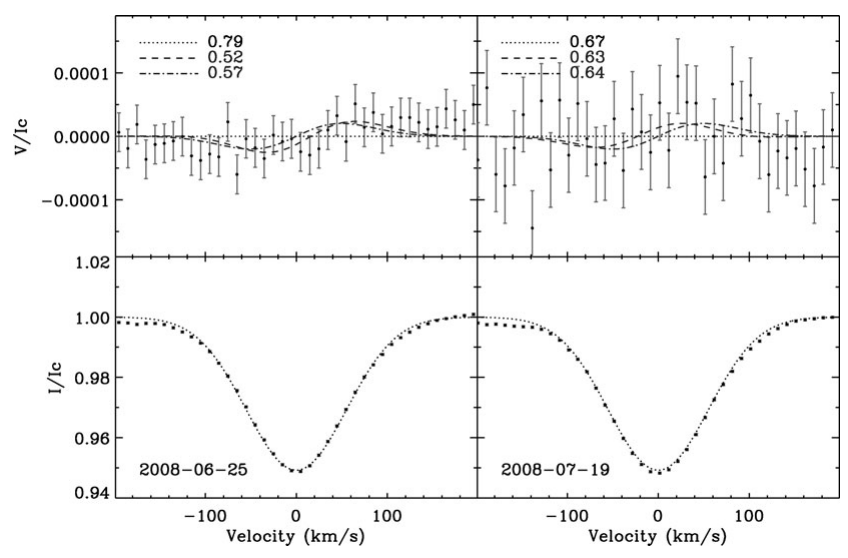

Figure 1. Lower: Mean Stokes I absorption line profiles of HD 155806 obtained with ESPaDOnS. Upper: Stokes V profiles. The best fit of a dipole model to an individual observation is shown in dashed lines and its corresponding likelihood is labelled, while the best fit to both observations is shown in dot-dashes lines. Formally, the individual fits give $\left\langle B_{z}\right\rangle=(-55 \pm 19,8 \pm 37) \mathrm{G}$, but neither is more significant than the fit for the null hypothesis of "no field" (dotted line).

\section{Observations and Analysis}

We used CFHT/ESPaDOnS to obtain high S/N spectropolarimetric observations of HD 155806 on 2008-06-25 and 2008-07-19. The lower panels of Fig. 1 show the mean absorption line profiles obtained with the least-squares deconvolution technique (Donati et al. 1997), while the upper panels show the distribution of circularly polarized light across the mean profiles. Since deviations as large as the ones observed would occur $(73 \%, 17 \%)$ of the time for the observations in (2008 June, 2008 July), these data provide no evidence for the presence of a magnetic field. In addition, our reanalysis of the 5 VLT/FORS1 grism observations discussed by Hubrig et al. (2008) resulted in nondetections. Thus, we cannot confirm the detection of a significant longitudinal field in HD 155806. A Bayesian analysis of the ESPaDoNs data shows that a dipolar magnetic field cannot be stronger than $(199,425) \mathrm{G}$ with a confidence of $(63.3,95.4) \%$.

\section{Implications}

For HD 155806, a wind magnetic confinement parameter of $\eta_{\star} \equiv B_{\text {eq }}^{2} R_{\star}^{2} / \dot{M} v_{\infty} \geqslant 1$ (ud-Doula \& Owocki 2002) implies that a dipolar field of at least $235 \mathrm{G}$ is required to confine the wind. Since upper limits on the non-detection of a magnetic field in the ESPaDOnS spectra are comparable, we conclude that the disk of HD 155806 is not caused by magnetic confinement of its wind. Similarly, Nazé, et al. (2010) did not detect the hard X-ray component in HD 155806 that should be produced in such a hot star by the collision of channelled streams of wind emerging from opposite magnetic hemispheres. Evidently the disk signatures in the spectrum of HD 155806 require a different explanation.

\section{References}

Donati, J.-F., Semel, M., Carter, B. D., Rees, D. E. et al. 1997, MNRAS, 291, 658

Hubrig, S., Yudin, R. V., Pogodin, M., Schöller, M. et al. 2007, AN, 328, 1133

Hubrig, S., Schöller, M., Schnerr, R. S., González, J. F. et al. 2008, A\&̈A, 490, 793

Nazé, Y., Rauw, G., \& Ud-Doula, A. 2010, A\&A, 510A, 59

ud-Doula, A. \& Owocki, S. P. 2002, ApJ, 576, 413

Walborn, N. R. 1973, AJ, 78, 1067 